\title{
Phosphorus is an independent risk factor for the progression of diabetic nephropathy
}

\author{
Haiyan Xiang ${ }^{A-D}$, Haitao Zhang ${ }^{A, E}$, Minlin Zhou' , Song Jiang ${ }^{C}$, \\ Lihua Zhang ${ }^{B}$, Dacheng Chen ${ }^{C}$, Zhihong Liu ${ }^{\mathrm{E}}$ \\ National Clinical Research Center of Kidney Diseases, Jinling Hospital, Nanjing University School of Medicine, China \\ A - research concept and design; $\mathrm{B}$ - collection and/or assembly of data; $\mathrm{C}$ - data analysis and interpretation; \\ $D$ - writing the article; $E$ - critical revision of the article; $F$ - final approval of the article
}

Address for correspondence

Haitao Zhang

E-mail: htzhang136@163.com

Funding sources

None declared

\section{Conflict of interest}

None declared

\section{Acknowledgements}

All samples were collected from the Renal Biobank of National Clinical Research Center of Kidney Diseases. This work was supported by the National Key Technology R\&D Program (2013BAI09B04, 2015BAI12B05), the Jiangsu Provincial clinical science and technology project (BL2012007) and the Innovation Capability Development Project of Jiangsu Province (BM2015004).

Received on November 15, 2016

Reviewed on April 1, 2017

Accepted on April 2, 2017

\section{Abstract}

Background. Serum phosphorus is thought to be an important risk factor for the progression of chronic kidney disease (CKD). However, the association of serum phosphorus with disease progression in patients with different causes of kidney diseases remains to be elucidated.

Objectives. The aim of this study was to estimate the effect of serum phosphorus on disease progression in 2 cohorts of CKD with different causes.

Material and methods. A total of 591 patients with diabetic nephropathy and 957 patients with IgA nephropathy from the National Clinical Research Center of Kidney Diseases, Nanjing, China, with biopsy-proven kidney disease, stage 1-4CKD and a follow-up of at least 1 year were recruited. We evaluated the relationship between the baseline phosphorus category and the disease progression in the 2 cohorts.

Results. Multivariate Cox regression analyses indicated that the risk of the endpoint event was 1.68-fold higher (95\% confidence interval (Cl): 0.95-2.91) in IgA nephropathy patients and 2.88-fold higher (95\% Cl: $1.12-5.04)$ in diabetic nephropathy patients with the highest quartile of serum phosphorus compared with the risk of those with the lowest quartile.

Conclusions. The association of serum phosphorus with the progression of CKD may vary in specific CKD patient subgroups. Serum phosphorus is independently associated with the progression of kidney disease in patients with diabetic nephropathy.

Key words: risk factors, chronic kidney disease, phosphorus, IgA nephropathy, diabetic nephropathy

DOI

10.17219/acem/70094

\section{Copyright}

Copyright by Author(s)

This is an article distributed under the terms of the

Creative Commons Attribution Non-Commercial License

(http://creativecommons.org/licenses/by-nc-nd/4.0/) 


\section{Introduction}

Chronic kidney disease (CKD) has become a major worldwide public health issue due to its increasing prevalence, poor outcomes and high cost of treatment for kidney failure. ${ }^{1}$ Early diagnosis and proper management of CKD and its risk factors are crucial for slowing the progression of the disease. In addition to the common risk factors of CKD progression, a growing body of evidence has suggested a link between serum phosphorous and the progression of CKD. ${ }^{2}$

Hyperphosphatemia is associated with an increased rate of hyperparathyroidism, mineral and bone disorder (MBD), vascular calcifications, cardiovascular events and mortality, and it has been demonstrated to be an independent risk factor for the progression of CKD. ${ }^{2,3}$ However, some studies have failed to reveal such a relationship between serum phosphorous and the progression of CKD. ${ }^{4}$ Notably, most of the existing data concerning the relationship between serum phosphorus and the prognosis of CKD did not include a sufficiently long follow-up period, and comparisons between different underlying kidney diseases have remained unclear. Some studies have shown differences in phosphorus metabolism in patients with diabetic nephropathy vs non-diabetic nephropathy;however, the relationship between serum phosphorus and CKD progression is unclear. ${ }^{5,6}$

We studied 2 cohorts that were treated at our center from August 2003, including patients with biopsy-positive IgA nephropathy and diabetic nephropathy with stage 1-4 CKD. ${ }^{7,8}$ We investigated the association of higher serum phosphorus with disease progression in patients with different causes of kidney disease.

\section{Material and methods}

\section{Patients and data collection}

All the samples and data were collected from the Renal Biobank of the National Clinical Research Center of Kidney Diseases, Nanjing, China. The study protocols were approved by the Ethical Committee of Jinling Hospital, Nanjing, China. Two cohorts of diabetic nephropathy and IgA nephropathy with stage 1-4 CKD according to the 2012 Kidney Disease: Improving Global Outcomes (KDIGO) clinical practice guidelines for CKD from the renal division of Nanjing Jinling Hospital were enrolled from August 2003 to October 2014. ${ }^{9}$ All patients were biopsy-diagnosed and regularly followed up at our facility. The diagnostic criteria for diabetic nephropathy and IgA nephropathy were described in 2 previous studies. ${ }^{7,8}$ The exclusion criteria included patients with the following characteristics: 1. an estimated glomerular filtration rate (eGFR) of $<15 \mathrm{~mL} /$ $\min / 1.73 \mathrm{~m}^{2}$, receiving dialysis or kidney transplant; 2 . age of $<18$ years; 3 . followed for less than 1 year; 4. with incomplete medical records. Baseline clinical and laboratory parameters were collected, including general characteristics (e.g., age, gender, primary disease, history of diabetes mellitus and hypertension) and medication history (e.g., angiotensin-converting enzyme inhibitors - ACEIs, angiotensin II receptor blockers - ARBs, sodium bicarbonate, calcium, activated vitamin D, steroids, diuretics, or lipid-lowering drugs). All participants provided 12-h-fasting blood samples for laboratory tests (serum calcium and phosphorus, intact parathyroid hormones (iPTH), blood glucose, total cholesterol, triglycerides, highdensity lipoprotein cholesterol, low-density lipoprotein cholesterol, urea, creatinine, uric acid, albumin, routine urinalysis, and urine sediment) and 24-h urine samples for a proteinuria test. Two BP measurements were taken using a mercury sphygmomanometer after $5 \mathrm{~min}$ of rest, and the average was used in the analyses. Hypertension was defined as a systolic blood pressure (BP) of $\geq 140 \mathrm{~mm} \mathrm{Hg}$, a diastolic BP of $\geq 90 \mathrm{~mm} \mathrm{Hg}$ and/or the use of antihypertensive medications; body mass index (BMI) was calculated based on weight and height (weight $[\mathrm{kg}] /$ height $\left[\mathrm{m}^{2}\right]$ ).

We estimated eGFR using the Chronic Kidney Disease Epidemiology Collaboration (CKD-EPI) 2009 creatinine equation. ${ }^{10}$

Patients received regular follow-up care 2-4 times per year in the outpatient ward and the information collected at each visit was recorded. During the followup period, we maintained the patient's BP in the normal range; glycated hemoglobin $\left(\mathrm{HbA}_{1 \mathrm{c}}\right)$ was maintained at about $6.5 \%$ in patients with diabetes. End points were validated by at least 2 physicians. The endpoint events included the following: a sustained doubling of creatinine, an eGFR of $<15 \mathrm{~mL} / \mathrm{min} / 1.73 \mathrm{~m}^{2}$, and dialysis for more than 3 months or transplantation.

\section{Statistical analysis}

All data was analyzed using the statistical software SPSS 19.0 (SPSS Inc., Chicago, USA) and Stata/SE software v. 12 (Stata Corp, College Station, USA). Normally distributed variables are expressed as mean $\pm \mathrm{SD}$ and non-parametric variables are expressed as medians (ranges or interquartile ranges). Differences between the 2 groups were analyzed by Student's t-test (normally distributed, homogeneity of variance), the Welch-Satterthwaite t-test (normally distributed, heterogeneity of variance), or the Wilcoxon rank sum test (non-normally distributed). Differences between more than 2 groups were analyzed by analysis of variance (ANOVA) for continuous variables. Categorical variables are expressed as percentages and were compared using the Pearson's $\chi^{2}$ test. All p-values were 2-tailed, and values $<0.05$ were considered statistically significant. Patients were grouped into 4 strands based on the quartile of serum phosphorus at baseline. Time-to-event survival analysis using Cox proportional hazard modeling was performed to evaluate the relationship between serum phosphorus and the endpoint event. 


\section{Results}

General clinical and laboratory data at baseline is shown in Tables 1 and 2. A total of 591 patients were diagnosed with diabetic nephropathy. The median follow-up period was 40 months, and the average eGFR was $73.3 \pm 30.6 \mathrm{~mL} /$ $\mathrm{min} / 1.73 \mathrm{~m}^{2}$. IgA nephropathy was diagnosed in $957 \mathrm{pa}$ tients; additionally, the median follow-up period for this group was 83 months, and the average eGFR was $84.7 \pm 32.9 \mathrm{~mL} / \mathrm{min} / 1.73 \mathrm{~m}^{2}$. Patients with diabetic nephropathy were older, had a higher level of serum phosphorus and a higher incidence of hypertension.

At baseline, participants with higher serum phosphorus levels tended to have higher proteinuria, higher cholesterol, higher uric acid, lower eGFRs, and lower albumin. Additionally, participants with higher serum phosphorus in IgA nephropathy had higher triglyceride levels, and patients with diabetic nephropathy had a higher incidence of hypertension.

During the follow-up period, 208 participants (35.2\%) with diabetic nephropathy and 166 patients (17.3\%) with IgA nephropathy reached the endpoint. The incidence rates of reaching endpoint were 25 (95\% confidence interval (CI): $18,32)$ and 88 (95\% CI: 70, 102) per 1,000 person-years for patients with IgA nephropathy and diabetic nephropathy, respectively. Patients were grouped into 4 strands based on the quartile of serum phosphorus at baseline. In the highest phosphorus quartile group, $26.8 \%$ of IgA nephropathy patients and $57.7 \%$ of diabetic nephropathy patients reached the endpoint; these were significantly higher percentages than those of patients in the lowest quartile group. Participants in the higher serum phosphorus quartile groups had a higher cumulative incidence of endpoint events (log-rank $\mathrm{p}<0.001$; Fig. 1).

In diabetic nephropathy patients, univariate Cox regression revealed that the risk of experiencing an endpoint event was 5.99 times higher (95\% CI: 3.51-10.23; p < 0.001) in patients in the highest phosphorus quartile group than in patients in the lowest quartile group. After adjusting for age, gender, the presence of hypertension, albumin, total cholesterol, triglycerides, high-density lipoprotein cholesterol, low-density lipoprotein cholesterol, urea, calcium, uric acid, BMI, iPTH, proteinuria, and eGFR, multivariate Cox regression indicated that the risk of reaching an endpoint event was 2.88 times higher (95\% CI: 1.12-5.04; $\mathrm{p}=0.024)$ in the highest quartile group.

In IgA nephropathy patients, univariate Cox regression indicated that the risk of an endpoint event was 2.78 times higher (95\% CI: $1.75-4.43$; $\mathrm{p}<0.001)$ for patients in the

Table 1. Baseline characteristics of patients with IgA nephropathy by quartiles of serum phosphorus

\begin{tabular}{|c|c|c|c|c|c|}
\hline \multirow{3}{*}{ Parameter } & \multicolumn{4}{|c|}{ Phosphorus category } & \multirow{3}{*}{$p$-value } \\
\hline & quartile 1 & quartile 2 & quartile 3 & quartile 4 & \\
\hline & $n=239(25.0 \%)$ & $n=217(22.7 \%)$ & $n=262(27.3 \%)$ & $n=239(25.0 \%)$ & \\
\hline Serum phosphorus [mmol/L] & $0.9 \pm 0.1$ & $1.1 \pm 0.1$ & $1.2 \pm 0.1$ & $1.4 \pm 0.1$ & $<0.001$ \\
\hline Age [years] & $38.8 \pm 9.2$ & $37.2 \pm 9.3$ & $35.3 \pm 9.0$ & $34.2 \pm 9.7$ & $<0.001$ \\
\hline Male, n (\%) & $160(66.9)$ & $106(48.8)$ & $134(51.1)$ & $134(56.1)$ & 0.065 \\
\hline Hypertension, n (\%) & $106(44.4)$ & $96(44.2)$ & $104(39.7)$ & $119(49.8)$ & 0.134 \\
\hline $\mathrm{BMI}\left[\mathrm{kg} / \mathrm{m}^{2}\right]$ & $24.7 \pm 3.5$ & $24.8 \pm 2.7$ & $24.2 \pm 3.6$ & $23.9 \pm 3.1$ & 0.092 \\
\hline $\mathrm{eGFR}\left[\mathrm{mL} / \mathrm{min} / 1.73 \mathrm{~m}^{2}\right]$ & $85.5 \pm 30.5$ & $88.5 \pm 30.3$ & $86.5 \pm 33.7$ & $78.5 \pm 35.8$ & 0.007 \\
\hline $\begin{array}{l}\text { eGFR stage, n (\%) } \\
\geq 90 \\
60-89 \\
45-59 \\
30-44 \\
15-29\end{array}$ & $\begin{array}{c}120(25.6) \\
55(25.5) \\
33(26.2) \\
24(26.4) \\
7(12.5)\end{array}$ & $\begin{array}{c}117(25.0) \\
49(22.7) \\
27(21.4) \\
18(19.8) \\
6(10.7)\end{array}$ & $\begin{array}{l}132(28.2) \\
65(30.1) \\
25(19.8) \\
20(21.9) \\
20(35.7)\end{array}$ & $\begin{array}{l}99(21.2) \\
47(21.7) \\
41(32.6) \\
29(31.9) \\
23(41.1)\end{array}$ & $\begin{array}{c}0.142 \\
0.089 \\
<0.001 \\
<0.001 \\
<0.001\end{array}$ \\
\hline Creatinine [mg/dL] & $1.1 \pm 0.5$ & $1.1 \pm 0.5$ & $1.2 \pm 0.6$ & $1.3 \pm 0.7$ & $<0.001$ \\
\hline Urea nitrogen [mg/dL] & $16.1 \pm 5.8$ & $16.6 \pm 6.9$ & $17.2 \pm 8.5$ & $20.7 \pm 12.1$ & $<0.001$ \\
\hline Uric acid $[\mu \mathrm{mol} / \mathrm{L}]$ & $376.7 \pm 101.6$ & $365.5 \pm 107.6$ & $392.7 \pm 115.1$ & $407.3 \pm 118.6$ & $<0.001$ \\
\hline Albumin [g/L] & $41.2 \pm 5.2$ & $41.1 \pm 5.1$ & $40.1 \pm 5.3$ & $38.7 \pm 6.3$ & $<0.001$ \\
\hline Total cholesterol [mmol/L] & $4.9 \pm 1.4$ & $4.7 \pm 1.2$ & $4.9 \pm 1.3$ & $5.3 \pm 1.9$ & 0.002 \\
\hline Triglyceride [mmol/L] & $2.0 \pm 1.5$ & $1.6 \pm 0.9$ & $1.7 \pm 1.1$ & $1.8 \pm 1.5$ & 0.003 \\
\hline Calcium [mmol/L] & $2.2 \pm 0.1$ & $2.2 \pm 0.2$ & $2.2 \pm 0.2$ & $2.2 \pm 0.1$ & 0.964 \\
\hline Proteinuria [g/24 h] & $1.1 \pm 0.9$ & $1.2 \pm 1.1$ & $1.2 \pm 1.1$ & $1.5 \pm 1.2$ & $<0.001$ \\
\hline Fasting blood glucose [mmol/L] & $5.1 \pm 1.3$ & $5.3 \pm 1.5$ & $4.9 \pm 1.8$ & $5.2 \pm 1.6$ & 0.526 \\
\hline LDL-cholesterol [mmol/L] & $3.5 \pm 1.8$ & $3.8 \pm 1.5$ & $3.2 \pm 1.3$ & $3.5 \pm 1.2$ & 0.213 \\
\hline HDL-cholesterol [mmol/L] & $1.0 \pm 0.8$ & $0.9 \pm 0.5$ & $1.1 \pm 0.9$ & $1.1 \pm 0.8$ & 0.329 \\
\hline iPTH [pg/mL] & $42.9 \pm 32.8$ & $54.3 \pm 32.2$ & $88.1 \pm 64.5$ & $190.9 \pm 103.6$ & $<0.001$ \\
\hline
\end{tabular}

BMI - body mass index; eGFR - estimated glomerular filtration rate; LDL - low density lipoprotein; HDL - high density lipoprotein; iPTH - intact parathyroid hormone. 
Table 2. Baseline characteristics of patients with diabetic nephropathy by quartiles of phosphorus

\begin{tabular}{|c|c|c|c|c|c|}
\hline \multirow{3}{*}{ Parameter } & \multicolumn{4}{|c|}{ Phosphorus category } & \multirow{3}{*}{$\mathrm{p}$-value } \\
\hline & quartile 1 & quartile 2 & quartile 3 & quartile 4 & \\
\hline & $n=145(24.5 \%)$ & $n=141(23.9 \%)$ & $n=156(26.4 \%)$ & $n=149(25.2 \%)$ & \\
\hline Serum phosphorus [mmol/L] & $1.0 \pm 0.1$ & $1.2 \pm 0.1$ & $1.3 \pm 0.1$ & $1.5 \pm 0.2$ & $<0.001$ \\
\hline Age [years] & $50.0 \pm 8.3$ & $50.5 \pm 8.9$ & $48.9 \pm 9.0$ & $48.3 \pm 10.5$ & 0.192 \\
\hline Male, n (\%) & $98(67.5)$ & $96(68.1)$ & $98(62.8)$ & $88(59.0)$ & 0.073 \\
\hline Hypertension, n (\%) & $101(69.7)$ & $112(79.4)$ & $124(79.5)$ & $127(85.2)$ & 0.012 \\
\hline $\mathrm{BMI}\left[\mathrm{kg} / \mathrm{m}^{2}\right]$ & $26.1 \pm 3.5$ & $25.9 \pm 3.7$ & $25.2 \pm 3.5$ & $25.1 \pm 3.3$ & 0.059 \\
\hline eGFR $\left[\mathrm{mL} / \mathrm{min} / 1.73 \mathrm{~m}^{2}\right]$ & $84.2 \pm 24.1$ & $80.6 \pm 30.3$ & $69.5 \pm 29.9$ & $59.7 \pm 28.5$ & $<0.001$ \\
\hline $\begin{array}{l}\text { eGFR stage, n (\%) } \\
\geq 90 \\
60-89 \\
45-59 \\
30-44 \\
15-29\end{array}$ & $\begin{array}{l}71(33.5) \\
42(28.2) \\
15(15.6) \\
14(15.2) \\
3(7.3)\end{array}$ & $\begin{array}{l}65(30.7) \\
37(24.8) \\
16(16.7) \\
17(18.2) \\
6(14.6)\end{array}$ & $\begin{array}{l}50(23.5) \\
35(23.5) \\
31(32.3) \\
27(29.0) \\
13(31.7)\end{array}$ & $\begin{array}{l}26(12.3) \\
35(23.5) \\
34(35.4) \\
35(37.6) \\
19(46.3)\end{array}$ & $\begin{array}{l}<0.001 \\
<0.001 \\
<0.001 \\
<0.001 \\
<0.001\end{array}$ \\
\hline Creatinine $[\mathrm{mg} / \mathrm{dL}]$ & $1.1 \pm 0.4$ & $1.1 \pm 0.5$ & $1.3 \pm 0.6$ & $1.5 \pm 0.6$ & $<0.001$ \\
\hline Urea nitrogen [mg/dL] & $18.7 \pm 6.5$ & $20.7 \pm 8.6$ & $22.6 \pm 8.8$ & $29.0 \pm 11.8$ & $<0.001$ \\
\hline Uric acid $[\mu \mathrm{mol} / \mathrm{L}]$ & $350.5 \pm 98.1$ & $372.3 \pm 96.0$ & $381.9 \pm 99.8$ & $401.8 \pm 101.8$ & $<0.001$ \\
\hline Albumin $[\mathrm{g} / \mathrm{L}]$ & $44.0 \pm 5.7$ & $41.7 \pm 7.6$ & $38.7 \pm 7.3$ & $37.0 \pm 7.1$ & $<0.001$ \\
\hline Total cholesterol [mmol/L] & $4.9 \pm 1.2$ & $5.5 \pm 2.2$ & $5.7 \pm 1.9$ & $6.0 \pm 1.8$ & 0.003 \\
\hline Triglyceride $[\mathrm{mmol} / \mathrm{L}]$ & $2.2 \pm 0.9$ & $2.3 \pm 1.3$ & $2.0 \pm 1.1$ & $2.0 \pm 1.1$ & 0.389 \\
\hline Calcium $[\mathrm{mmol} / \mathrm{L}]$ & $0.8 \pm 0.4$ & $0.9 \pm 0.5$ & $0.9 \pm 0.5$ & $0.9 \pm 0.6$ & 0.207 \\
\hline Proteinuria [g/24 h] & $1.8 \pm 1.3$ & $2.3 \pm 1.7$ & $2.5 \pm 1.5$ & $2.8 \pm 1.9$ & $<0.001$ \\
\hline Fasting blood glucose $[\mathrm{mmol} / \mathrm{L}]$ & $7.2 \pm 1.6$ & $7.3 \pm 1.3$ & $6.9 \pm 1.8$ & $7.1 \pm 2.2$ & 0.264 \\
\hline LDL-cholesterol [mmol/L] & $3.4 \pm 1.9$ & $3.7 \pm 1.7$ & $3.5 \pm 1.3$ & $3.8 \pm 1.6$ & 0.286 \\
\hline HDL-cholesterol [mmol/L] & $1.1 \pm 0.3$ & $0.9 \pm 0.3$ & $1.1 \pm 0.5$ & $1.0 \pm 0.6$ & 0.516 \\
\hline $\mathrm{HbA}_{1 c}[\%]$ & $7.2 \pm 1.8$ & $7.3 \pm 1.5$ & $7.3 \pm 1.6$ & $7.4 \pm 1.2$ & 0.132 \\
\hline iPTH [pg/mL] & $50.9 \pm 23.8$ & $65.7 \pm 22.5$ & $96.1 \pm 52.3$ & $205.9 \pm 95.6$ & $<0.001$ \\
\hline
\end{tabular}

BMI - body mass index; eGFR - estimated glomerular filtration rate; LDL - low density lipoprotein; HDL - high density lipoprotein; HbA1c - hemoglobin A1c; PTH - intact parathyroid hormone.
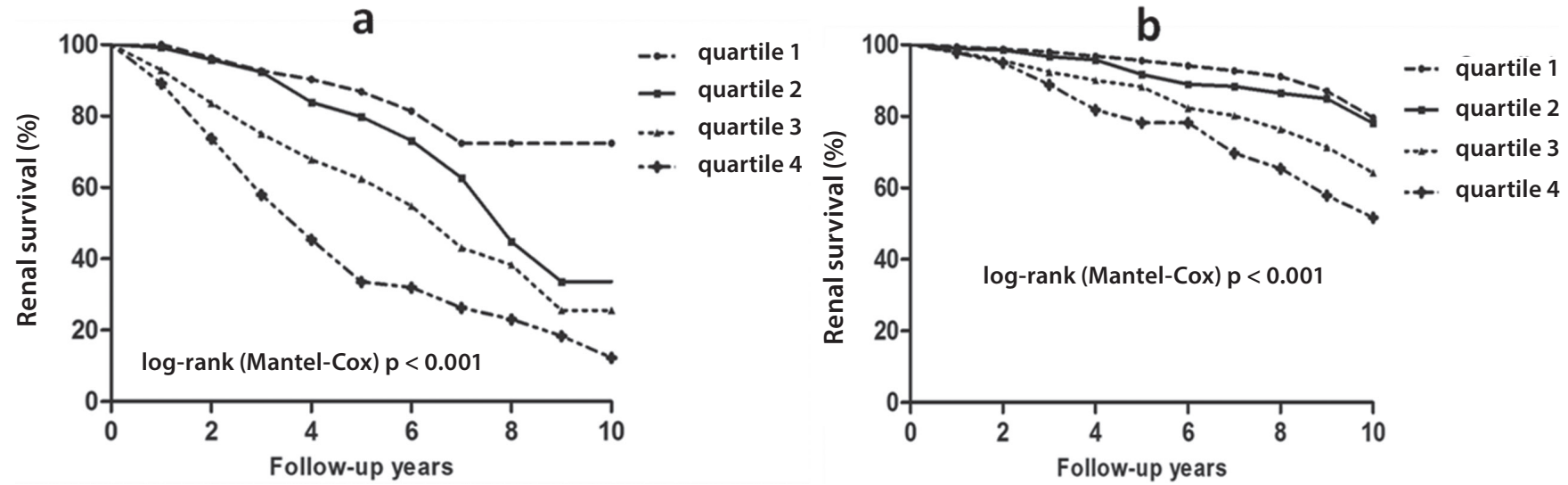

Fig. 1. Renal survival curves according to the quartile of serum phosphorus in the subgroups of CKD patients

A - diabetic nephropathy; B - IgA nephropathy.

highest phosphorus quartile group than for patients in the lowest quartile group. After adjusting for risk factors, the risk of reaching an endpoint was attenuated such that statistical significance was lost (HR 1.68; 95\% CI: 0.95-2.91; $\mathrm{p}=0.086$ ) (Table 3 ).

Subgroup multivariate Cox analysis was performed for patients with an eGFR of more or less than $60 \mathrm{~mL} /$
$/ \mathrm{min} / 1.73 \mathrm{~m}^{2}$. In diabetic nephropathy patients, the highest quartile of phosphorous level predicted a greater risk of the endpoint (HR 5.37, (95\% CI: 1.61-12.26; p = 0.009)) in the eGFR $\geq 60 \mathrm{~mL} / \mathrm{min} / 1.73 \mathrm{~m}^{2}$ group, as shown in Fig. $2 \mathrm{~A}$. Multivariate Cox regression showed no correlation between serum phosphorus and the progression of kidney disease in IgA nephropathy, as shown in Fig. 2 B. 
Table 3. Cox regression of prognosis associated with baseline serum phosphorus in diabetic nephropathy and IgA nephropathy

\begin{tabular}{|c|c|c|c|c|}
\hline \multirow{2}{*}{ Serum phosphorus } & \multirow{2}{*}{$\begin{array}{c}\text { Diabetic nephropathy } \\
\text { HR }(95 \% \mathrm{Cl})\end{array}$} & \multirow[b]{2}{*}{$p$-value } & \multicolumn{2}{|c|}{ IgA nephropathy } \\
\hline & & & $\mathrm{HR}(95 \% \mathrm{CI})$ & $p$-value \\
\hline \multicolumn{5}{|c|}{ Unadjusted } \\
\hline Quartile 1 (ref) & 1 & & 1 & \\
\hline Quartile 2 & $2.47(1.37,4.45)$ & 0.003 & $1.05(0.61,1.81)$ & 0.85 \\
\hline Quartile 3 & $4.17(2.42,7.17)$ & $<0.001$ & $1.87(1.16,3.01)$ & 0.01 \\
\hline Quartile 4 & $5.99(3.51,10.23)$ & $<0.001$ & $2.78(1.75,4.43)$ & $<0.001$ \\
\hline \multicolumn{5}{|c|}{ Multivariate adjusted } \\
\hline Quartile 1 (ref) & 1 & & 1 & \\
\hline Quartile 2 & $1.81(0.82,4.02)$ & 0.143 & $0.95(0.59,1.76)$ & 0.638 \\
\hline Quartile 3 & $2.14(1.02,4.47)$ & 0.044 & $1.52(0.85,2.72)$ & 0.098 \\
\hline Quartile 4 & $2.88(1.12,5.04)$ & 0.024 & $1.68(0.95,2.91)$ & 0.082 \\
\hline
\end{tabular}

a

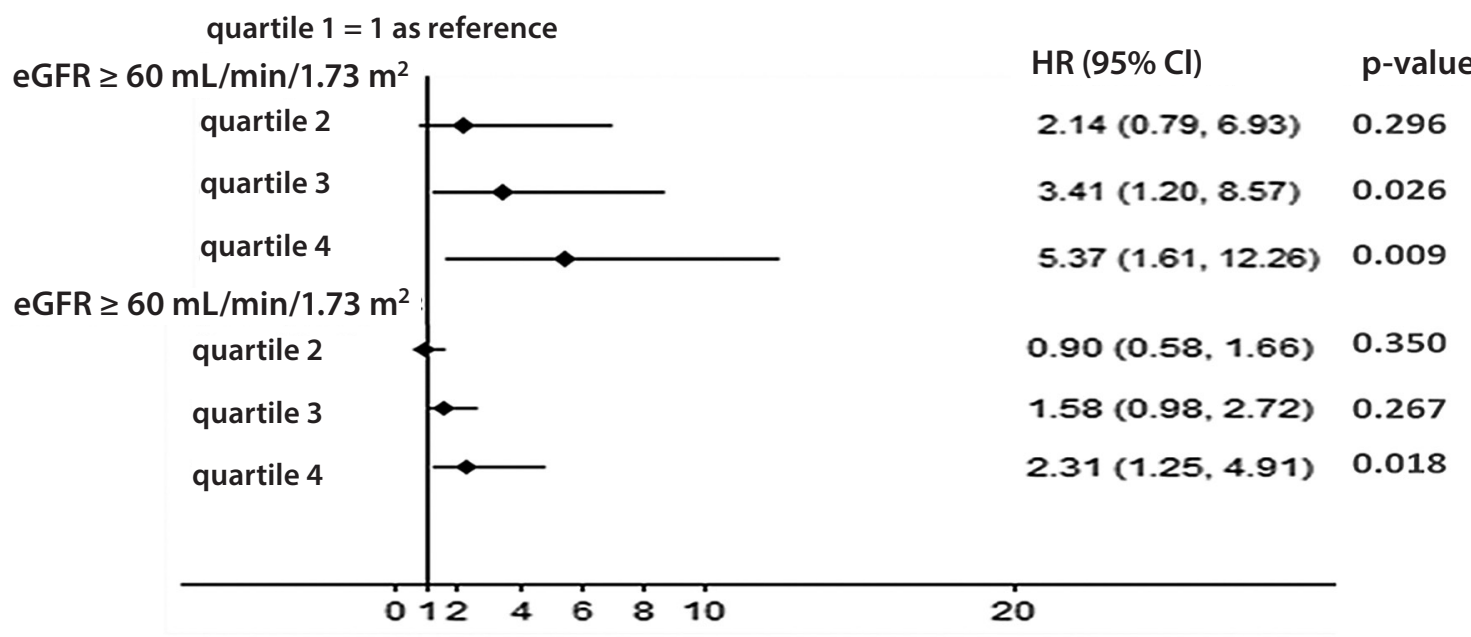

b

quartile $1=1$ as reference

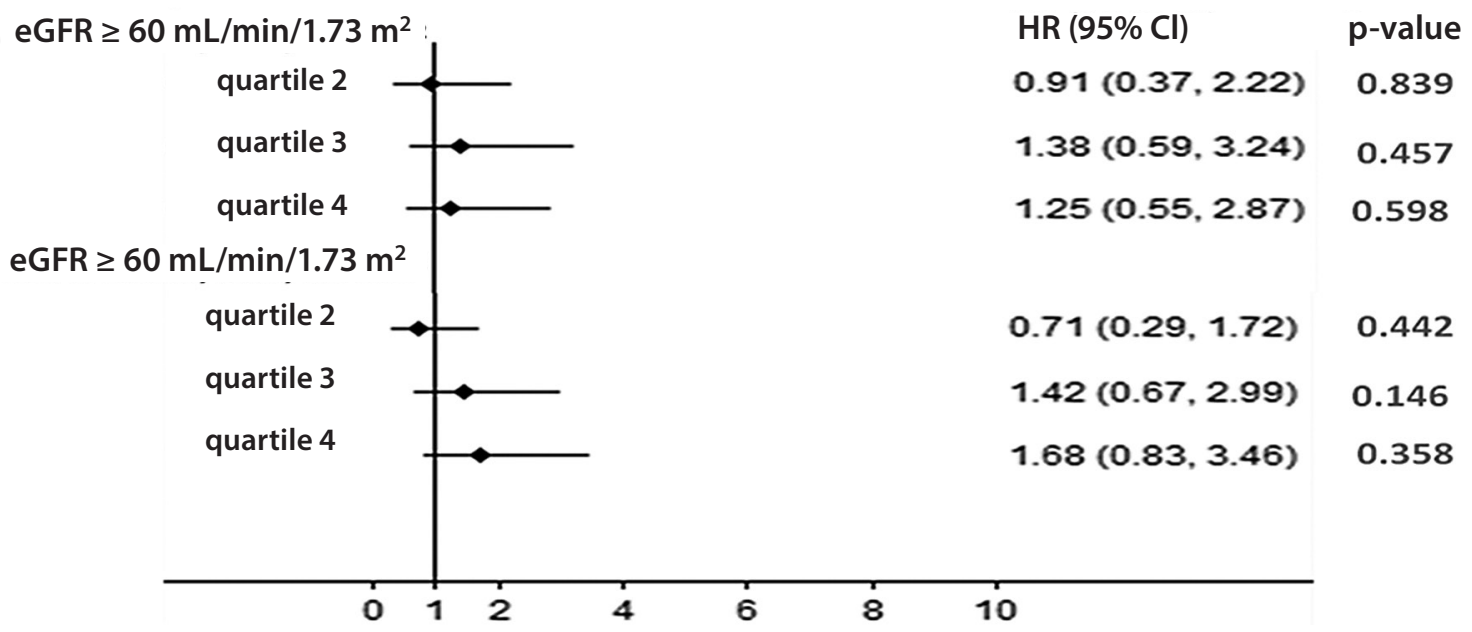

Fig. 2. Subgroup analysis of the association between serum phosphorus and disease progression 


\section{Discussion}

Hyperphosphatemia is a major complication of CKD and is negatively correlated with residual renal function and prognosis. The proper management of serum phosphorus is critical at all stages of CKD. ${ }^{11}$ Hyperphosphatemia has been reported to be associated with an increased risk of disease progression, end stage renal disease (ESRD) and a rapid deterioration of residual renal function. ${ }^{12-15}$ However, Mehrotra et al. failed to find an association of serum phosphorus with CKD progression and ESRD in the Kidney Early Evaluation Program (KEEP) study. ${ }^{4}$ Because the median follow-up period of this study was only 2.3 years, further research covering a much longer period is needed to confirm this conclusion.

The leading causes of CKD are chronic glomerulonephritis and diabetic nephropathy, and IgA nephropathy is the most common cause of chronic glomerulonephritis. Prognosis varies between different subgroups of CKD, among which diabetic nephropathy patients exhibit the most rapid progression. ${ }^{16,17}$ Thus, the etiologies of CKD should be considered when assessing the progression of CKD due to the notable differences in the progression of diabetic nephropathy compared with that of other diseases. The current study indicated that patients with IgA nephropathy had a more promising prognosis than patients with diabetic nephropathy. The risk factors for end-stage renal disease (ESRD) differed between the 2 cohorts in our previous studies, but the relationship between phosphorus and prognosis was not mentioned.,8

Previous studies revealed a significant difference in the diagnosis of mineral and bone disorder in diabetic patients compared with non-diabetic patients. Derangements of mineral metabolism began to occur earlier in patients with diabetes; in stage 1-2 CKD patients with diabetic nephropathy, elevated serum phosphorus and fibroblast growth factor 23 (FGF-23) levels and decreased calcium and vitamin D levels were detected. ${ }^{6,18,19}$ The current study demonstrated that the prevalence of hyperphosphatemia was higher among diabetic nephropathy patients compared with patients with IgA nephropathy in every stage of CKD, consistent with previous reports. Previous studies confirmed that diabetic nephropathy patients had lower urinary phosphate excretion and higher serum phosphate levels than non-diabetic nephropathy patients, and that increased FGF-23 and decreased vitamin D levels occurred earlier in diabetic nephropathy patients than in nondiabetic patients. ${ }^{16,17,20}$ Therefore, the prevalence of hyperphosphatemia is higher among patients with diabetic nephropathy compared with non-diabetic nephropathy. ${ }^{6}$

Many studies have explored the association between serum phosphorus and CKD progression. Bellasi et al. showed higher predictive values of phosphorus for disease progression in diabetic nephropathy than non-diabetic nephropathy. ${ }^{21}$ Fliser et al. found that FGF-23 was an independent risk factor for disease progression in the moderate and advanced stages of CKD in non-diabetic patients, while serum phosphorus had no predictive value (HR 1.09; $\mathrm{p}=0.063$ ). ${ }^{22}$ Such a discrepancy may be attributed to patient ethnicity and differing etiologies of CKD. The current study demonstrated that the association of serum phosphorus with CKD progression varied in different patient subgroups. Serum phosphorus was an independent risk factor for CKD progression in patients with diabetic nephropathy but was not an independent risk factor in patients with IgA nephropathy, indicating that serum phosphorus has a higher predictive value in patients with diabetic nephropathy than in patients with non-diabetic nephropathy.

The association of serum phosphorus with the progression of kidney disease in specific CKD patient subgroups remains to be elucidated. Animal models have demonstrated that hyperphosphatemia can lead to deteriorated renal function and renal structural alterations. ${ }^{23}$ Consumption of a high-phosphorus diet has been shown to accelerate the deterioration of renal function, and a low-phosphorus diet can delay the progression of CKD. ${ }^{24,25}$ Hyperphosphatemia can injure podocytes and compromise the glomerular filtration barrier by affecting pituitary-specific positive transcription factor 1 (Pit-1), resulting in glomerular sclerosis. ${ }^{26}$ In non-dialysis CKD patients, serum phosphorus was associated with increased levels of inflammatory cytokines and C-reactive protein. ${ }^{27}$ An increased phosphorus load results in systemic vascular endothelial injury and promotes the deposition of calcium and phosphorus in the renal tubule and the renal interstitial area, leading to tubular atrophy, interstitial fibrosis and a further decline in renal function. ${ }^{28}$ Thus, serum phosphorus is linked to adverse effects on renal function and prognosis in diabetic nephropathy, independent of other traditional risk factors, which may result from hormone imbalances regulated by phosphorous and the early presence of hyperphosphatemia. Increased phosphorus could further accelerate renal tubular dysfunction, inflammation, glomerular sclerosis, and renal function deterioration, but the exact mechanism remains to be identified.

It is widely recognized that phosphorus starts to accumulate in stage $3 \mathrm{~b}$ of CKD and begins to rise until eGFR decreases to $<40 \mathrm{~mL} / \mathrm{min} / 1.73 \mathrm{~m}^{2} .{ }^{29}$ However, the adverse health effects of hyperphosphatemia and/or an overload of phosphorus do not appear to be limited to the advanced stages of CKD. ${ }^{2,3}$ Previous reports have demonstrated in the early stages of CKD that serum phosphorus begins to accumulate and that elevations in serum phosphorus are associated with the risk of CKD progression. ${ }^{21}$ This study demonstrated that in the eGFR $\geq 60 \mathrm{~mL} / \mathrm{min} / 1.73 \mathrm{~m}^{2}$ group of patients with diabetic nephropathy, the highest quartile of phosphorous level predicted a greater risk of the endpoint. The result is also only found in diabetic nephropathy but not in IgA nephropathy.

The current study preliminarily analyzed the association of serum phosphorus with prognosis in specific CKD 
patient subgroups. Patients were monitored for an extended period of time. However, this study has several limitations that require discussion. Firstly, because this was a retrospective study, the influence of different treatment options - which would inevitably have had an impact on prognosis - was not investigated. Secondly, because few patients had repeated measurements of serum phosphorus, we used single baseline serum phosphorus measurements in analyses. Thirdly, factors that regulate calcium and phosphorus metabolism - including FGF-23 and vitamin D - were not detected, though they may be important mediators of the relationship between serum phosphorus and CKD progression.

\section{Conclusions}

The association of serum phosphorus with the progression of kidney disease may vary in specific CKD patient subgroups. Serum phosphorus is an independent risk factor for the progression of CKD in patients with diabetic nephropathy, even in the early stages of CKD. Thus, levels of serum phosphorus should be detected and well-controlled in the early stages of diabetic nephropathy in order to slow the progression of the disease.

\section{References}

1. Eckardt KU, Coresh J, Devuyst O, et al. Evolving importance of kidney disease: From subspecialty to global health burden. Lancet. 2013; 382(9887):158-169.

2. Da J, Xie X, Wolf M, et al. Serum phosphorus and progression of CKD and mortality: A meta-analysis of cohort studies. Am J Kidney Dis. 2015;66(2):258-265.

3. O'Seaghdha CM, Hwang SJ, Muntner $\mathrm{P}$, et al. Serum phosphorus predicts incident chronic kidney disease and end-stage renal disease. Nephrol Dial Transplant. 2011;26(9):2885-2890.

4. Mehrotra R, Peralta CA, Chen SC, et al. No independent association of serum phosphorus with risk for death or progression to end-stage renal disease in a large screen for chronic kidney disease. Kidney Int. 2013;84(5):989-997.

5. Schwarz S, Trivedi BK, Kalantar-Zadeh K, Kovesdy CP. Association of disorders in mineral metabolism with progression of chronic kidney disease. Clin J Am Soc Nephrol. 2006;1(4):825-831.

6. Wahl P, Xie H, Scialla J, et al. Earlier onset and greater severity of disordered mineral metabolism in diabetic patients with chronic kidney disease. Diabetes Care. 2012;35(5):994-1001.

7. Le $\mathrm{W}$, Liang $\mathrm{S}, \mathrm{Hu} \mathrm{Y}$, et al. Long-term renal survival and related risk factors in patients with IgA nephropathy: Results from a cohort of 1155 cases in a Chinese adult population. Nephrol Dial Transplant. 2012;27(4):1479-1485.

8. An Y, Xu F, Le W, et al. Renal histologic changes and the outcome in patients with diabetic nephropathy. Nephrol Dial Transplant. 2015; 30(2):257-266

9. Stevens PE, Levin A; Kidney Disease: Improving Global Outcomes Chronic Kidney Disease Guideline Development Work Group Members. Evaluation and management of chronic kidney disease: Synopsis of the kidney disease: Improving global outcomes 2012 clinical practice guideline. Ann Intern Med. 2013;158(11):825-830.
10. Levey AS, Stevens LA, Schmid $\mathrm{CH}$, et al. A new equation to estimate glomerular filtration rate. Ann Intern Med. 2009;150(9):604-612.

11. Slatopolsky E, Moe S. 50 years of research and discovery in chronic kidney disease and mineral \& bone disorder: The central role of phosphate. Kidney Int. 2011;79(S121):S1-S2.

12. Norris KC, Greene T, Kopple J, et al. Baseline predictors of renal disease progression in the African American study of hypertension and kidney disease. J Am Soc Nephrol. 2006;17(10):2928-2936.

13. Scialla JJ, Astor BC, Isakova T, Xie H, Appel LJ, Wolf M. Mineral metabolites and CKD progression in African Americans. J Am Soc Nephrol. 2013;24(1):125-135.

14. Chue CD, Edwards NC, Davis LJ, Steeds RP, Townend JN, Ferro CJ. Serum phosphate but not pulse wave velocity predicts decline in renal function in patients with early chronic kidney disease. Nephrol Dial Transplant. 2011;26(8):2576-2582.

15. Block GA, Ix JH, Ketteler M, et al. Phosphate homeostasis in CKD: Report of a scientific symposium sponsored by the National Kidney Foundation. Am J Kidney Dis. 2013;62(3):457-473.

16. Haynes R, Staplin N, Emberson J, et al. Evaluating the contribution of the cause of kidney disease to prognosis in CKD: Results from the study of heart and renal protection (SHARP). Am J Kidney Dis. 2014;64(1):40-48.

17. Ekart R, Ferjuc A, Furman B, Gerjevič Š, Bevc S, Hojs R. Chronic kidney disease progression to end stage renal disease: A single center experience of the role of the underlying kidney disease. Ther Apher Dial. 2013;17(4):363-367.

18. Tanaka $\mathrm{H}$, Hamano $\mathrm{T}$, Fujii $\mathrm{N}$, et al. The impact of diabetes mellitus on vitamin D metabolism in predialysis patients. Bone. 2009;45(5): 949-955.

19. Mehrotra R. Disordered mineral metabolism and vascular calcification in nondialyzed chronic kidney disease patients. J Ren Nutr. 2006;16(2):100-118.

20. Chen H, Li X, Yue R, Ren X, Zhang X, Ni A. The effects of diabetes mellitus and diabetic nephropathy on bone and mineral metabolism in T2DM patients. Diabetes Res Clin Pract. 2013;100(2):272-276.

21. Bellasi A, Mandreoli M, Baldrati L, et al. Chronic kidney disease progression and outcome according to serum phosphorus in mild-to-moderate kidney dysfunction. Clin J Am Soc Nephrol. 2011;6(4):883-891.

22. Fliser D, Kollerits B, Neyer U, et al. Fibroblast growth Factor 23 (FGF23) predicts progression of chronic kidney disease: The Mild to Moderate Kidney Disease (MMKD) study. J Am Soc Nephrol. 2007;18(9):2600-2608.

23. Neves KR, Graciolli FG, dos Reis LM, Pasqualucci CA, Moysés RM, Jorgetti V. Adverse effects of hyperphosphatemia on myocardial hypertrophy, renal function, and bone in rats with renal failure. Kidney Int. 2004;66(6):2237-2244.

24. Newsome $B, I x ~ J H, T i g h i o u a r t ~ H$, et al. Effect of protein restriction on serum and urine phosphate in the Modification of Diet in Renal Disease (MDRD) study. Am J Kidney Dis. 2013;61(6):1045-1046.

25. Isakova T, Barchi-Chung A, Enfield G, et al. Effects of dietary phosphate restriction and phosphate binders on FGF23 levels in CKD. Clin J Am Soc Nephrol. 2013;8(6):1009-1018.

26. Navarro-González JF, Mora-Fernández C, Muros M, Herrera H, García J. Mineral metabolism and inflammation in chronic kidney disease patients: A cross-sectional study. Clin J Am Soc Nephrol. 2009;4(10): 1646-1654.

27. Nadkarni GN, Uribarri J. Phosphorus and the kidney: What is known and what is needed. Adv Nutr. 2014;5(1):98-103.

28. Kanbay M, Goldsmith D, Akcay A, Covic A. Phosphate - the silent stealthy cardiorenal culprit in all stages of chronic kidney disease: A systematic review. Blood Purif. 2009;27(2):220-230.

29. Levin A, Bakris GL, Molitch M, et al. Prevalence of abnormal serum vitamin D, PTH, calcium, and phosphorus in patients with chronic kidney disease: Results of the study to evaluate early kidney disease. Kidney Int. 2007;71(1):31-38. 